\title{
The scavenger receptor repertoire in six cnidarian species and its putative role in cnidarian-dinoflagellate symbiois
}

\author{
Emilie F Neubauer $^{1}{ }^{\text {, Angela Z Poole }}{ }^{2}$, Virginia M Weis ${ }^{\text {Corresp.. }}{ }^{3}$, Simon K Davy ${ }^{1}$ \\ ${ }^{1}$ School of Biological Sciences, Victoria University of Wellington, Wellington, New Zealand \\ 2 Department of Biology, Western Oregon University, Monmouth, Oregon, United States \\ 3 Department of Integrative Biology, Oregon State University, Corvallis, Oregon, United States \\ Corresponding Author: Virginia M Weis \\ Email address: weisv@oregonstate.edu
}

Many cnidarians engage in a mutualism with endosymbiotic photosynthetic dinoflagellates that forms the basis of the coral reef ecosystem. Interpartner interaction and regulation includes involvement of the host innate immune system. Basal metazoans, including cnidarians have diverse and complex innate immune repertoires that are just beginning to be described. Scavenger receptors (SR) are a diverse superfamily of innate immunity genes that recognize a broad array of microbial ligands and participate in phagocytosis of invading microbes. The superfamily includes subclades named SR-A through SR-I that are categorized based on the arrangement of sequence domains including the scavenger receptor cysteine rich (SRCR), the C-type lectin (CTLD) and the CD36 domains. Previous functional and gene expression studies on cnidarian-dinoflagellate symbiosis have implicated SR-like proteins in interpartner communication and regulation. In this study, we characterized the SR repertoire from a combination of genomic and transcriptomic resources from six cnidarian species in the Class Anthozoa. We combined these bioinformatic analyses with functional experiments using the SR inhibitor fucoidan to explore a role for SRs in cnidarian symbiosis and immunity. Bioinformatic searches revealed a large diversity of SR-like genes that resembled SR-As, SR-Bs, SR-Es and SR-Is. SRCRs, CTLDs and CD36 domains were identified in multiple sequences in combinations that were highly homologous to vertebrate SRs as well as in proteins with novel domain combinations. Phylogenetic analyses of CD36 domains of the SR-B-like sequences from a diversity of metazoans grouped cnidarian with bilaterian sequences separate from other basal metazoans. All cnidarian sequences grouped together in a subclade separately from bilaterian sequences with moderate support. Functional experiments were carried out on the sea anemone Aiptasia pallida that engages in a symbiosis with Symbiodinium minutum (clade B1). Experimental blocking of the SR ligand binding site with the inhibitor fucoidan reduced the ability of $S$. minutum to colonize Aiptasia suggesting that host SRs play a role 
in host-symbiont recognition. In addition, incubation of symbiotic anemones with fucoidan elicited an immune response, indicating that host SRs function in immune modulation that results in host tolerance of the symbionts. 
1 The scavenger receptor repertoire in six cnidarian species and its putative role in

2 cnidarian-dinoflagellate symbiosis

3

4 Emilie F. Neubauer ${ }^{1}$, Angela Z. Poole ${ }^{2}$, Virginia M. Weis ${ }^{3}$, Simon K. Davy ${ }^{1}$

5

$6 \quad{ }^{1}$ School of Biological Sciences, Victoria University of Wellington, Wellington, New Zealand 7

${ }^{2}$ Department of Biology, Western Oregon University, Monmouth, Oregon, USA

${ }^{3}$ Department of Integrative Biology, Oregon State University, Corvallis, Oregon, USA

Corresponding Authors:

Virginia Weis

weisv@oregonstate.edu

Simon Davy

Simon.Davy@vuw.ac.nz 


\section{Abstract}

31

32

33

34

35

36

37

38

39

40

41

42

43

44

45

46

47

48

49

50

51

52

53

54

55

56

57

58

Many cnidarians engage in a mutualism with endosymbiotic photosynthetic dinoflagellates that forms the basis of the coral reef ecosystem. Interpartner interaction and regulation includes involvement of the host innate immune system. Basal metazoans, including cnidarians have diverse and complex innate immune repertoires that are just beginning to be described. Scavenger receptors (SR) are a diverse superfamily of innate immunity genes that recognize a broad array of microbial ligands and participate in phagocytosis of invading microbes. The superfamily includes subclades named SR-A through SR-I that are categorized based on the arrangement of sequence domains including the scavenger receptor cysteine rich (SRCR), the Ctype lectin (CTLD) and the CD36 domains. Previous functional and gene expression studies on cnidarian-dinoflagellate symbiosis have implicated SR-like proteins in interpartner communication and regulation. In this study, we characterized the SR repertoire from a combination of genomic and transcriptomic resources from six cnidarian species in the Class Anthozoa. We combined these bioinformatic analyses with functional experiments using the SR inhibitor fucoidan to explore a role for SRs in cnidarian symbiosis and immunity. Bioinformatic searches revealed a large diversity of SR-like genes that resembled SR-As, SR-Bs, SR-Es and SR-Is. SRCRs, CTLDs and CD36 domains were identified in multiple sequences in combinations that were highly homologous to vertebrate SRs as well as in proteins with novel domain combinations. Phylogenetic analyses of CD36 domains of the SR-B-like sequences from a diversity of metazoans grouped cnidarian with bilaterian sequences separate from other basal metazoans. All cnidarian sequences grouped together with moderate support in a subclade separately from bilaterian sequences. Functional experiments were carried out on the sea anemone Aiptasia pallida that engages in a symbiosis with Symbiodinium minutum (clade B1). Experimental blocking of the SR ligand binding site with the inhibitor fucoidan reduced the ability of S. minutum to colonize $A$. pallida suggesting that host SRs play a role in host-symbiont recognition. In addition, incubation of symbiotic anemones with fucoidan elicited an immune response, indicating that host SRs function in immune modulation that results in host tolerance of the symbionts. 
60

61

62

63

64

65

66

67

68

69

70

71

72

73

74

75

76

77

78

79

80

81

82

83

84

85

86

87

88

89

90

91

92

93

94

95

96

97

98

\section{Introduction}

Cnidarians such as reef-building corals engage in an intimate mutualistic symbiosis with photosynthetic dinoflagellates in the genus Symbiodinium that together form the trophic and structural foundation of coral reef ecosystems. Symbiodinium spp. provide large amounts of reduced organic carbon to the host in exchange for inorganic nutrients, a high light environment and refuge from herbivory (Yellowlees et al. 2008). In the majority of cnidarian-Symbiodinium interactions, the symbionts are taken up by host cells via phagocytosis. Instead of being digested as food, the symbionts resist host destruction and persist in host cells by residing in vacuoles known as symbiosomes (Davy et al. 2012). The molecular interplay between host cnidarian and resident symbionts during both the establishment and ongoing maintenance of the symbiosis is critical for a healthy holobiont (Weis \& Allemand 2009).

Animal innate immune systems are central to managing microbes by both tolerating and promoting the survival of beneficial symbionts and resisting and destroying negative invaders (Bordenstein \& Theis 2015; McFall-Ngai et al. 2013; Schneider \& Ayres 2008). With the increased availability of sequence resources, there is now ample evidence that innate immune pathways are ancestral and that basal metazoans including cnidarians possess many of these pathways originally described in mammals and flies (Fuess et al. 2016; Miller et al. 2007; Yuen et al. 2014). Furthermore there are numerous examples of expansions of some innate immune gene families in invertebrates that are larger than those in vertebrate genomic repertoires, including NOD-like receptors, scavenger receptors, TIR-domain-containing proteins and ficolins (Baumgarten et al. 2015; Buckley \& Rast 2015; Hamada et al. 2013; Pancer 2000; Poole \& Weis 2014; Shinzato et al. 2011). A class of well-described host-microbe molecular interactions mediated by innate immunity are the PRR-MAMP interactions where microbe-associated molecular patterns (MAMPs) on the surface of microbes, such as lipopolysaccharide or glycans, are recognized by pattern recognition receptors (PRRs) on the surface of host cells (Janeway \& Medzhitov 2002). These steric interactions launch a series of downstream signalling cascades in the host that serve to resist and destroy negative invaders or tolerate and nurture positive microbes. Genomic and transcriptomic studies of cnidarians are revealing the presence of many classical PRRs that have been extensively characterized in higher metazoans (Fuess et al. 2016; Miller et al. 2007).

One group of PRRs in the Metazoa are the scavenger receptors (SRs), so-named for their role in the scavenging and clearing of microbial invaders, modified host molecules, and apoptotic cell debris (Areschoug \& Gordon 2009; Canton et al. 2013). SRs have a high affinity for a wide range of ligands and this flexibility of ligand binding has led them to be described as 'molecular fly paper' (Krieger 1992). A key role of SRs in innate immune function is their action as PRRs on phagocytic cells where they mediate direct non-opsonic phagocytosis of pathogenic microbes (Areschoug \& Gordon 2009). SRs are thought to engage in heteromultimeric signalling 
99 complexes, known as signalosomes, involving multiple PRRs and other molecules that together

100 effect signal transduction in cells, thereby alerting them to microbes or modified host molecules

101 (Canton et al. 2013). The SR superfamily is a large group of structurally diverse transmembrane

102 cell surface glycoproteins, divided into nine classes SR-A through SR-I (Canton et al. 2013;

103 Krieger 2001). The classes have overlapping specificities that result in an enormous breadth of

104 MAMP recognition (Krieger 1992). Members within a given class share some sequence

105 homology, with little-to-no homology occurring between classes. The classes are grouped by

106 their multiple domains with no single domain common to all (Gordon 2002; Gough \& Gordon

107 2000). SR domains occur on the extracellular portion of the protein; the proteins are anchored in

108 the cell membrane with transmembrane domain(s) and contain short cytoplasmic tail(s). Figure 1

109 depicts the four SR classes that are relevant to this study. SRs are a potential target for

110 manipulation by invading parasites, pathogens and potentially mutualists. Several pathogens

111 have evolved mechanisms to evade SR-mediated recognition (Areschoug \& Waldemarsson

112 2008; Faure \& Rabourdin-Combe 2011). Indeed, certain human pathogens exploit specific SRs

113 for their own benefit. For example, the Hepatitis C virus (HCV) (Catanese et al. 2007) and the

114 malaria parasite Plasmodium falciparum (Ndungu et al. 2005; Rodrigues et al. 2008) have

115 surface ligands that are recognized by SR-B1, and both use this recognition to gain entry to host

116 cells.

117

118 SR-As and SR-Is contain the scavenger receptor cysteine rich (SRCR) domain, which consists of

119 a 110 aa motif with conserved spacing of six to eight cysteines (Hohenester et al. 1999). The

120 SRCR domain is found in a wide range of membrane and soluble proteins and often occurs in

121 multiple repeats arrayed on the protein (Hohenester et al. 1999; Martinez et al. 2011; Sarrias et

122 al. 2004). Some SR-As and SR-Es contain C-type lectin domains (CTLDs), a common domain in

123 many proteins, that are often involved in lectin-glycan interactions (Cambi et al. 2005). SR-Bs

124 contain the CD36 domain and have two cytoplasmic tails rooted in the membrane with two

125 transmembrane regions, forming an extracellular loop (Silverstein \& Febbraio 2009). SR genes

126 encoding SRCR, CTLD and CD36 domains have been described in invertebrates (Hibino et al.

127 2006; Lehnert et al. 2014; Pancer et al. 1997; Schwarz et al. 2007; Wood-Charlson \& Weis

128 2009). However, a detailed bioinformatic characterization of cnidarian SR genes homologous to

129 vertebrate SR-As, SR-Bs, SR-Es and SR-Is is lacking, as are any functional studies exploring the

130 function of these proteins.

131

132 SRs are of interest in studies of cnidarian immunity and symbiosis. First, interactions between

133 SR-E-like host lectin-like proteins and symbiont surface glycans play an important role in host-

134 symbiont recognition during onset of symbiosis (reviewed in Davy et al. 2012). In addition, SR-

135 B homologues in two species of sea anemone, Anthopleura elegantissima (Rodriguez-Lanetty et

136 al. 2006) and Aiptasia pallida (Lehnert et al. 2014), were found to be highly expressed in

137 symbiotic compared to aposymbiotic individuals. For $A$. pallida this was a dramatic difference in

138 expression where symbiotic anemones had 28-fold greater expression than aposymbiotic 
139 animals. These studies suggest that SR-E and SR-B homologues are playing a role in host-

140 symbiont communication.

141

142 There were two aims for this study. The first was to identify SRs in six cnidarian species, all in

143 Class Anthozoa (corals, sea anemones and others), using a variety of genomic and transcriptomic

144 resources, and compare the repertoire to vertebrate SRs of known function. This provides a

145 platform for identifying potential roles of cnidarian SR proteins in immunity and symbiosis. The

146 second aim was to perform simple functional experiments to examine the role of SRs in

147 symbiont recognition and uptake by the sea anemone $A$. pallida, a well-studied model system for

148 the study of coral-dinoflagellate symbiosis. We hypothesized that if a symbiont is co-opting host

149 SRs to initiate tolerogenic pathways that dampen or prevent an immune response, blocking SR-

150 ligand-binding capabilities would induce an immune response. 


\section{Materials and Methods}

153

154

155 To characterize the SR protein repertoire in cnidarians, six species with publically available

156 resources were searched. These included three anemone species: A. elegantissima (Kitchen et al.

157 2015), Aiptasia pallida (Baumgarten et al. 2015; Lehnert et al. 2012), and Nematostella vectensis

158 (Putnam et al. 2007), and three coral species: Acropora digitifera (Shinzato et al. 2011), $A$.

159 millepora (Moya et al. 2012) and Fungia scutaria (Kitchen et al. 2015). These species were

160 selected based on the availability of transcriptomic and genomic resources, and to include a

161 diversity of organisms within Class Anthozoa. These resources were derived from various

162 developmental stages and symbiotic states (Table 1). All resources were used as provided, with

163 the exception of the $A$. pallida transcriptome, for which raw Illumina sequence reads for

164 accession SRR696721 were downloaded from the sequence read archive entry

165 (http://www.ncbi.nlm.nih.gov/sra/SRX231866) and reassembled using Trinity (Grabherr et al.

166 2011) resulting in a better assembly than the original one performed.

167

168

SR sequence searching

169 Twenty-four non-cnidarian sequences were obtained, primarily from GenBank and other

170 publically available databases (Table S1), for use in creating multiple sequence alignments and

171 phylogenetic trees. Eleven human SR genes were chosen for production of reference protein

172 domain architecture diagrams, to compare predicted cnidarian proteins with human SR proteins

173 of known function (Figure 2).

To search for cnidarian SR proteins, databases were queried using several search strategies to ensure all sequences were recovered. BLASTp or tBLASTn searches with mouse and human SR protein sequences (SR-A1, MARCO, SRCL, CD36, SRB1, LMP2, and LOX1) (Supplementary file 1) and consensus sequences (pfam01130: CD36, pfam00530: SRCR) from the conserved domain database (http://www.ncbi.nlm.nih.gov/cdd) as queries were performed for each resource. Keyword searches were used with the terms SR, CD36, LMP2, SRCR, and scavenger where GO or KEGG annotations were available. Lastly, representative $N$. vectensis sequences of each protein type (SRCR-domain-containing, CD36, SRB1, and LOX1) were also used as queries for tBLASTn searches of the other five cnidarian resources. A high e-value cutoff $\left(1 \times 10^{-}\right.$ ${ }^{1}$ ) was used in the BLAST searches to recover divergent sequences. All BLAST searches were performed using the default settings in Geneious pro version 7.1.8 with the exception of $N$. vectensis, for which searches were performed through the Joint Genome Institute online portal using the default settings (Kearse et al. 2012). A list of metazoan resources searched are listed in Table S1. Blast query sequences and cnidarian sequences identified are tabulated in

189 Supplementary File 1.

190 
191 To confirm that the sequences obtained contained SR domains, nucleotide sequences were

192 translated using Geneious and then annotated using the InterProScan plugin (Quevillon et al.

193 2005). Only sequences in which two or more databases within InterProScan found either SRCR,

194 CD36, or CTLD domains with an e-value of $<1 \times 10^{-4}$ were used. Where the InterProScan plugin

195 was unable to resolve protein domains, (this occurred for approx. 1 in 10 sequences) the

196 sequences were analysed using the online protein domain database PfamA

197 (http://pfam.sanger.ac.uk) using the default program settings. Sequences for each species were

198 aligned and those that were identical or almost identical $(<5$ aa difference in the conserved

199 domains) were omitted from the analysis as they likely represented artifacts of assembly or

200 different isoforms of the same protein. Sequences missing a start or stop codon were removed

201 from the analysis.

202

203

Only proteins that showed significant PfamA matches to a CTLD, SRCR and/or CD36 domains

204 were included in the study. Diagrammatic representations of the protein domain configurations

205 were produced using this information. Protein domain architectures were grouped together

206

207

208 according to common domains and compared to known human SR proteins (Figure 2).

Phylogenetic analysis of SR-B homologues

209

A multiple sequence alignment of a subset of CD36-domain-containing sequences was

210 performed with the MAFFT v 7.017 plug-in (Katoh et al. 2002) through Geneious (Kearse et al. 2012), using the default settings. The program ProtTest v2.4 (Abascal et al. 2005) was used to

212

213 apply AIC1, AIC2 and BIC2 model selection criteria to a variety of possible substitution matrices and rate assumptions to obtain the best-fit model of protein evolution. The results from the overall comparison of these metrics indicated that the best-fit model for the full-length alignment was $\mathrm{WAG}+\mathrm{G}+\mathrm{F}$. A maximum likelihood tree was produced using FastTree v2.1.5 (Price et al. 2010). Bootstrap support values were generated using the online program SEQBOOT (Felsenstein 2005) and values above 0.6 support were displayed at the nodes. A PhyML (Guindon et al. 2005) alternate tree produced identical topology (data not shown).

\section{Maintenance and preparation of anemone and dinoflagellate cultures}

222 Symbiotic A. pallida cultures were maintained in saltwater aquaria at $26^{\circ} \mathrm{C}$ with a $12 / 12 \mathrm{~h}$

223 light/dark photoperiod, and were fed twice weekly with live brine shrimp nauplii. Animals were rendered aposymbiotic by incubation for $8 \mathrm{~h}$ at $4{ }^{\circ} \mathrm{C}$ twice weekly for six weeks, followed by maintenance in the dark for approximately one month. Anemones were fed twice weekly with brine shrimp, and cleaned of expelled symbionts and food debris regularly. maintained in $50 \mathrm{ml}$ flasks in sterile Guillard's f/2 enriched seawater culture medium (Sigma, St. Louis, MO, USA). Dinoflagellate cultures were maintained at $26^{\circ} \mathrm{C}$ on a $12 / 12 \mathrm{~h} \mathrm{light/dark}$ 230 photoperiod. 
232 In preparation for experimental manipulations, individual anemones were placed in 24-well

233

234

235

236

237

238

239

240

241

242

243

244

245

246

247

248

249

250

251

252

253

254

255

256

257

258

259

260

261

262

263

264

265

266

267

268

269

270 plates in $2.5 \mathrm{ml}$ of $1-\mu \mathrm{m}$ filtered seawater (FSW) and acclimated to the well-plate for 3-4 days, with the FSW replaced daily. Well plates containing aposymbiotic anemones were kept in the dark and symbiotic anemones were maintained in an incubator at $26^{\circ} \mathrm{C}$ with a $12 / 12 \mathrm{~h}$ light/dark photoperiod. Animals were not fed during the experimental time period.

\section{Addition of fucoidan to block SR binding function}

To explore a role for SRs in the onset of symbiosis, fucoidan, a known SR ligand, was added to anemones to block SR binding sites. Fucoidan is a protein derived from the brown alga Fucus vesiculosus; this polyanionic ligand is known to bind SRCR and CD36 domains in SR-As and SR-Bs, respectively (Dinguirard \& Yoshino 2006; Hsu et al. 2001; Thelen et al. 2010).

To examine the effect of blocking SR ligand binding capabilities on symbiont colonization success, aposymbiotic anemones $(n=3$ per treatment per time point) were pre-incubated in fucoidan (Sigma, St. Louis, MO, USA), at a concentration of 0 (FSW control), 100, 200 and 400 $\mu \mathrm{g} / \mathrm{ml}$ for $18 \mathrm{~h}$, according to Bowdish Lab protocols (online at McMaster University; www.bowdish.ca/lab/protocols). Fucoidan-treated aposymbiotic anemones were subsequently reinoculated with $S$. minutum CCMP830. CCMP830 cells were pelleted from the culture medium, re-suspended in FSW, and then added to anemones in well-plates to a final concentration of $2 \times 10^{5}$ symbionts per $\mathrm{ml}$. After incubation for $12 \mathrm{~h}$ at $26^{\circ} \mathrm{C}$ in the light, anemones were rinsed twice with FSW and fucoidan treatments were refreshed. To test the effect of fucoidan exposure on host health, a second control treatment (fucoidan-washed control) was prepared where aposymbiotic anemones were pre-incubated in $200 \mu \mathrm{g} / \mathrm{ml}$ fucoidan for $18 \mathrm{~h}$, and then washed with FSW prior to being inoculated with symbionts as described above. Anemones for all treatments were sampled at 48 and $96 \mathrm{~h}$ post-infection (three tentacles per anemone, for $\mathrm{n}=3$ anemones per treatment per time point).

A second experiment was designed to explore a role for SR binding in host immune tolerance during symbiosis. We hypothesized that if a symbiont is co-opting host SRs to initiate tolerogenic pathways (such as the TGF $\beta$ pathway) that dampen or prevent an immune response, blocking SR-ligand-binding capabilities could induce an immune response upon the addition of lipopolysaccharide (LPS). LPS is a MAMP that has been shown to induce an anemone immune response measured as increased nitric oxide (NO) production (Detournay et al. 2012; Perez \& Weis 2006). Symbiotic anemones were incubated at increasing concentrations of fucoidan: 0 (FSW control), 100, 200, 400 and $800 \mu \mathrm{g} / \mathrm{ml}$, for $4 \mathrm{~h}$, prior to the addition of $1 \mu \mathrm{g} / \mathrm{ml}$ of LPS (Sigma, St. Louis, MO, USA) (dissolved in 0.1\% v/v DMSO) for a further $12 \mathrm{~h}$. The FSW control was also exposed to $1 \mu \mathrm{g} / \mathrm{ml}$ LPS for 12 hours. NO production by hosts was quantified as described below. 
271 Quantifying colonization success and host NO production using confocal microscopy

272 Colonization success was assessed fluorometrically by confocal microscopy, following methods

273 described in detail by Detournay et al (2012). Briefly, following experimental manipulation,

274 solutions in wells containing anemones were replaced with $1 \mathrm{ml}$ of relaxing solution $(1: 10.37 \mathrm{M}$

$275 \mathrm{MgCl}_{2}$ : FSW). Samples were observed under a Zeiss LSM 510 Meta microscope with a 40x/0.8

276 water objective lens and a working distance of 0.8-3.2 $\mathrm{mm}$. Before image scanning, the focal

277 plane of the optical section was adjusted to include the gastrodermal cells within the anemone

278 tentacle. For each experiment, all images were obtained with the same software scanning

279 settings, including detector gain and laser intensity. S. minutum cells present were visualized by

280 detecting chlorophyll autofluorescence with excitation and emission wavelengths of 543 and

$281600-700 \mathrm{~nm}$, respectively. Fluorescence was quantified by first defining the gastrodermal tissue

282 area within the anemone tentacles as a region of interest and then measuring the mean

283 fluorescence intensity (MFI) for that region with the LSM 5 software. Intensity of chlorophyll

284 autofluorescence for each pixel was measured and a threshold value corresponding to the

285 background was defined by measuring the MFI at $600 \mathrm{~nm}$ of a gastrodermal region without

286 symbionts (threshold MFI =20). Colonization success was expressed as percent of pixels with

287 autofluorescence intensity above the threshold. In colonization experiments, each treatment

288 represents a sample size of three anemones per treatment and time-point, with percent

289 colonization taken as the mean of six tentacles per anemone. Three untreated symbiotic

290 anemones (six tentacles per anemone) were examined to determine a baseline colonization level

291 for symbiotic anemones.

292

The statistical significance of colonization success under the treatments described above was

294

295 assessed using a mixed-effects model. As measures on multiple samples (i.e., tentacles) per anemone violate independence assumptions, a mixed effect was used, treating anemone as a random effect to account for correlation among samples with anemones. Main effects included time and treatment, and their interaction was estimated to account for differences between treatments at each time point. The full model can be written as:

299

300

301

302

303

Here, $y_{i, j}$ is the logarithm of percent colonization (plus a small constant) of tentacle $i$ within

304 anemone $j, \beta$ is a vector of effects to be estimated, $X$ is a design matrix encoding the treatment and time point, as well as interaction term contrasts, $\mu_{j}$ is a normally distributed random effect for anemone $j$, and $\epsilon_{i, j}$ are normally distributed residuals. The model was estimated using the LME4 packages (Bates et al. 2015), for the statistical computing software R (R-Core-Team 2012) (www.R-project.org), the script and data used for statistical analyses are given in Supplementary Files 2, 3 and 4. 
311 To measure and visualize production of NO by confocal microscopy, animals were treated as 312 described in detail previously (Detournay et al. 2012; Detournay \& Weis 2011). Animals were 313 transferred to a microfuge tube containing $500 \mu \mathrm{l}$ of relaxing solution and $15 \mu \mathrm{M} 4$-amino-5314 methylamino-2,7 difluorofluorescein diacetate (DAF-FM DA, Molecular Probes, Eugene, OR, 315 USA) with excitation and emission wavelengths of 488 and $510-530 \mathrm{~nm}$, respectively. Samples

316 were incubated for $30 \mathrm{~min}$ in the dark and then rinsed twice with relaxing solution. Fluorescence 317 of the DAF-FM DA probe was quantified as described above for chlorophyll autofluorescence 318 quantification.

319

320 The statistical analysis of this experiment used a similar model as above, but treating the 321 fucoidan concentration as a continuous variable and fitting a linear slope to the fluorescence 322 intensity. We also added a random effect for tentacle within anemone to account for non323 independence of readings within each tentacle. Using the notation above, the model can be 324 written as

325

326

327

328 Here, $y_{k, i, j}$ is the fluorescence reading $k$ within tentacle $i$ of anemone $j, \alpha$ is the intercept and $\beta$ is 329 the slope of the regression line relating fluorescence to fucoidan concentration $F_{k}, \mu_{j}$ is a 330 normally distributed random effect for anemone $j, \gamma_{i(j)}$ is a normally distributed random effect 331 for tentacle $i$ within anemone $j$, and $\epsilon_{k, i, j}$ are normally distributed residuals.

332

333 


\section{Results}

335

336

Annotated predicted cnidarian SR proteins are illustrated according to their domain architecture

337 and compared with known human SR protein domain organization (Figure 2). Overall, cnidarian SR-like proteins fall into four groups: SR-As, SR-Es, SR-Is and SR-Bs. The SRCR domain is present in all groups except the SR-Bs.

340

\section{Cnidarian SRCR-containing proteins}

342 Vertebrate SR-As are defined by a collagen domain coupled with most proteins containing either

343 an SRCR domain or a CTLD at the C terminus (Bowdish \& Gordon 2009). Only two sequences

344 meeting these criteria were identified in the cnidarian resources searched. Both are in $A$.

345 digitifera and contain a CUB domain in addition to two collagen domains and one SRCR.

346 Human SR-Es are defined by the presence of only CTLDs (Zani et al. 2015). The human lectin-

347 like oxidized low-density lipoprotein receptor 1 (LOX1) has an N-terminal cytoplasmic tail, a

348 transmembrane domain and a single C-terminal CTLD (Canton et al. 2013). Numerous LOX1like sequences were identified in all of the cnidarian resources searched. SR-Is in humans are defined by containing only SRCR domains in various numbers of repeats and are grouped into three classes: CD5, CD6 and CD163. SR-I-like sequences are abundant in all cnidarian resources, in the same configurations as human SR-Is. SRCR repeat numbers range from one to twenty-three.

354

355

A variety of SRCR-domain-containing proteins were also identified in cnidarian sequence resources that could not be classified into any of the vertebrate classes of scavenger receptors. Several cnidarians genes with SRCRs and CUB domains were identified that resemble 'human deleted in malignant brain tumor' (DMBT) protein that contains eight SRCR repeats, a single CUB domain and a zona pelucida domain at the C-terminal end. Predicted cnidarian proteins that resemble DMBT contain one to three CUB domains combined with a range of other protein domains, including MAM, fibronectin UBOX and multiple SRCRs. Five of the six cnidarian resources contain sequences with a potentially novel domain configuration of multiple SRCRs, several other domains, including multiple immunoglobulin domains, and a C-terminal trypsin domain.

\section{Cnidarian SR-B-like proteins}

367 Searches identified eighteen full-length putative cnidarian SR-B sequences, all containing a

368 CD36 domain. Full-length proteins were defined as those containing both transmembrane

369 regions that form the SR-B extracellular loop configuration. Humans have four distinct SR-Bs -

370 CD36, SRB1 \& 2, and LMP2 - while the six cnidarian species searched contained between two and four full-length proteins.

372

373

\section{Phylogenetic analysis of SR-B-like proteins}


374 Phylogenetic analysis was carried out on the CD36 domains from SR-B-like sequences identified 375 (Figure S1 and Figure 3). Protein sequence alignments of the predicted SR-B-like proteins from 376 cnidarians, combined with a subset of vertebrate and invertebrate sequences, revealed that there 377 is some conservation of the CD36 domain across metazoans. Cnidarian sequences showed weak 378 homology to human SR-Bs, with $26-32 \%, 28-37 \%$ and $28-33 \%$ identity to human CD36, LMP2, 379 and SR-B1, respectively. Identities within the cnidarian group were higher, ranging from 39 to 95\%, with the two Acropora species showing the highest homology to each other. Cnidarian sequences showed between 21 and 27\% identity to the predicted SR-B-like protein sequence from the sponge, Suberites domuncula. Predicted cnidarian proteins lacked one of the three pairs of cysteine residues known to form three disulphide bridges in the human CD36 protein (Figure S1) (Silverstein and Febbraio, 2009). However, a pair of cysteine residues was found in all cnidarian study species at positions $\mathrm{C} 107$ and $\mathrm{C} 117$. Predicted cnidarian proteins had 8-10 Nlinked glycosylation sites compared with eleven and eight sites in human SR-B1 and CD36, respectively.

388

389

Putative cnidarian SR-B proteins grouped with high support in a large clade with the bilaterians and separately from other basal metazoans. Within this large clade, cnidarians grouped together, forming a separate clade from the bilaterians. Within the cnidarian clade, there were three wellsupported sub-clades, two containing both coral and anemone species and a third, containing only anemone sequences (Figure 3). Corals and sea anemones sequences formed distinct groupings within each of these clades. In contrast, bilaterian invertebrate sequences grouped with mammalian sequences in several different sub-clades of SR-Bs: LMP2, CD36, CD36-like, SRB1, and SR-B1-like proteins.

397

Experimental blocking of SR proteins with fucoidan reduces colonization success and elicits an immune response in $\mathrm{A}$. pallida Fucoidan-treated anemones showed significantly lower levels of colonization (0-3\%) than either the FSW control or anemones pre-incubated in fucoidan and then rinsed $48 \mathrm{~h}$ prior to time zero Colonization success decreased significantly in a dose-dependent manner (Figure 4, Bayesian $\mathrm{P}$ $<0.0001)$.

A second fucoidan experiment investigated the possible immune-regulation role of an SR in 406 symbiosis maintenance. Symbiotic anemones were treated with increasing concentrations of fucoidan and were subsequently immune-challenged by incubation with LPS. The FSW controltreated anemones had low levels of NO production, a proxy for an immune response, measured as MFI of the NO-specific probe DAF-FM DA in tentacles, in response to incubation in LPS. In contrast, fucoidan-treated anemones showed a significant (Bayesian $\mathrm{P}<0.0001$ ) dose-dependent

411 response of increasing NO production with increasing concentrations of fucoidan (Figure 5). 


\section{Discussion}

414

415 An expanded SRCR-domain-containing protein repertoire in cnidarians

416 The SRCR-domain-containing protein repertoire in cnidarians, is expanded compared to that in

417 humans, with the $A$. pallida genome containing the highest number at 36 genes (Figure 2). This

418 finding is consistent with numerous other studies describing expansions of innate immune gene

419 families in invertebrates (see Introduction). Other examples of SRCR-domain-containing protein

420 repertoire expansion have been described in invertebrates, specifically in the sea urchin,

421 Strongylocentrotus purpuratus and the cephalochordate Branchiostoma floridae, which have 218

422 and 270 SRCR-containing sequences respectively (Huang et al. 2008; Pancer 2000; Pancer et al.

423 1999; Rast \& Messier-Solek 2008). These numbers are high compared to the 16 genes present in

424 humans. In addition, cnidarian SRCR-domain-containing proteins include a variety of genes with

425 novel domain combinations that have not been found in other organisms (Figure 2).

426 Identification of these novel domain combinations in cnidarian immune gene repertoires is

427 consistent with other studies of basal metazoan immune genes (Hamada et al. 2013; Poole \&

428 Weis 2014; Ryu et al. 2016). The searches for SR genes in the three transcriptomes (Table 1)

429 likely revealed underestimates of the total SR repertoire, given that transcriptomes represent

430 snapshots of the whole genome.

431

432

\section{CTLD-domain-containing SRs in cnidarians}

433 In contrast to the human genome, which contains a single LOX1 gene, all six cnidarian resources

434 searched contained multiple LOX1-like SR-Es (Figure 2). These searches add to previous characterizations of lectin-like proteins in cnidarians, including in corals and sea anemones (Jimbo et al. 2005; Jimbo et al. 2000; Kvennefors et al. 2010; Kvennefors et al. 2008; Meyer \& Weis 2012; Vidal-Dupiol et al. 2009; Wood-Charlson \& Weis 2009). Human LOX1 has a diversity of signalling functions, including in recognition of microbes via host CTLD-microbe glycan binding: a PRR-MAMP interaction (Canton et al. 2013). In cnidarians, previous studies have detailed a role for lectin-glycan interactions in the establishment of cnidarian-dinoflagellate symbioses (reviewed in Davy et al. 2012). The identification of multiple LOX1-like proteins and several other CTLD-containing proteins with novel domain combinations across the six species examined further strengthens the hypothesis that host CTLD-symbiont glycan binding plays an important role in host innate immunity and host-symbiont recognition. Cnidarian CTLD-domaincontaining proteins described here provide potential target proteins for future experimental investigation of the lectin-glycan interactions.

\section{CD36-domain-containing SRs in cnidarians} Phylogenetic analysis of metazoan CD36 domains from SR-B homologues showed a wellsupported clade of cnidarian sequences (Figure 3). A large analysis including additional sequences from basal metazoans is required to more definitively reveal deep branching patterns of this gene. The observed differing location of cysteine pairs within the CD36 domain in 
454 S1). As with the cnidarians searched, C. elegans contained one differing pair and the three

455

456

457

458

459

460

461

462

463

464

465

466

467

468

469

470

471

472

473

474

475

476

477

478

479

480

481

482

483

484

485

486

487

488

489

490

491

492

sponges, Oscarella carmella, S. domuncula, and Amphimedon queenslandica, and the ctenophore Mnemiopsis leidyi had no sequence pairs in common with vertebrates. These differences may explain why antibodies to human and mouse SR-B1 and CD36 failed to label proteins in A. pallida in immunoblot experiments (E.F. Neubauer, unpublished data).

Functional experiments suggest that blocking SRs decreases colonization success and increases the stress response to immune challenge in A. pallida Colonization success in aposymbiotic $A$. pallida challenged with $S$. minutum CCMP830 displayed a dose-dependent response to incubation in the SR inhibitor fucoidan, exhibiting decreasing colonization success with increasing concentrations of fucoidan (Figure 4). In vertebrates, fucoidan blocks the positively-charged ligand binding sites on SR-As and SR-Bs, and can thereby block phagocytic activity in macrophages (Dinguirard \& Yoshino 2006; Hsu et al. 2001; Li et al. 2008). The observed inhibition of colonization in cnidarians suggests that phagocytosis of symbionts is likewise inhibited and provides evidence that one or multiple SRs with SRCR and/or CD36 domains function in host-symbiont recognition during onset of symbiosis.

Previous transcriptomic studies in A. elegantissima and A. pallida have found SR-B homologues to be upregulated in symbiotic compared to aposymbiotic anemones, suggesting that they play a role in the symbiosis. Our experiments showing that incubation in fucoidan causes a dosedependent immune response in symbiotic A. pallida (Figure 5), further implicates a role for SRs in immune tolerance and regulation of symbiosis. In previous work on A. pallida, we showed that symbiotic anemones produced significantly less NO in response to an immune challenge with LPS than did aposymbiotic animals, suggesting that symbionts are modulating the host immune response (Detournay et al., 2012). The increase in this response in symbiotic anemones incubated in fucoidan suggests that this immune modulation involves an SR ligand-binding domain. Such a response is reminiscent of immune modulation by a variety of invading microbes (Janeway \& Medzhitov 2002).

In summary, this study provides the first description of the diversity of SRs in cnidarians. Members include proteins with domain combinations that are highly similar to those in vertebrates as well as those that possess novel combinations. Initial functional experiments using the SR inhibitor fucoidan suggest that SRs play a role in the regulation of cnidariandinoflagellate symbioses. Future functional studies on candidate SRs identified in this study can further explore their role in cnidarian immunity and symbiosis. 


\section{Acknowledgements}

494

495 We thank Eli Meyer for the reassembly of the A. pallida transcriptome and Philipp Neubauer for 496 assistance with statistical analysis. We wish to acknowledge the Confocal Microscopy Facility at 497 the Center for Genome Research and Biocomputing at Oregon State University. 
498

499

500

501

502

503

504

505

506

507

508

509

510

511

512

513

514

515

516

517

518

519

520

521

522

523

524

525

526

527

528

529

530

531

532

533

534

535

536

537

538

539

540

541

542

\section{References}

Abascal F, Zardoya R, and Posada D. 2005. ProtTest: selection of best-fit models of protein evolution. Bioinformatics 21:2104-2105. DOI:

Areschoug T, and Gordon S. 2009. Scavenger receptors: role in innate immunity and microbial pathogenesis. Cellular Microbiology 11:1160-1169. DOI: 10.1111/j.14625822.2009.01326.x

Areschoug T, and Waldemarsson J. 2008. Evasion of macrophage scavenger receptor Amediated recognition by pathogenic Streptococci. EMBO Journal 38:3068-3079. DOI: 10.1002/eji.200838457

Bates D, Maechler M, Ben B, and Walker S. 2015. Fitting linear mixed-effects models using lme4. Journal of Statistical Software 67:1-48. DOI: 10.18637/jss.v067.i0

Baumgarten S, Simakov O, Esherick LY, Liew YJ, Lehnert EM, Michell CT, Li Y, Hambleton EA, Guse A, Oates ME, Gough J, Weis VM, Aranda M, Pringle JR, and Voolstra CR. 2015. The genome of Aiptasia, a sea anemone model for coral symbiosis. Proceedings of the National Academy of Sciences 112:11893-11898. DOI: 10.1073/pnas.1513318112

Bordenstein SR, and Theis KR. 2015. Host Biology in Light of the Microbiome: Ten Principles of Holobionts and Hologenomes. PLoS Biol 13:e1002226. DOI:

10.1371/journal.pbio.1002226

Bowdish DME, and Gordon S. 2009. Conserved domains of the class A scavenger receptors: evolution and function. Immunological Reviews 227:19-31. DOI: 10.1111/j.1600065X.2008.00728.X

Buckley KM, and Rast JP. 2015. Diversity of animal immune receptors and the origins of recognition complexity in the deuterostomes. Developmental and Comparative Immunology 49:179-189. DOI: 10.1016/j.dci.2014.10.013

Cambi A, Koopman M, and Figdor CG. 2005. How C-type lectins detect pathogens. Cellular Microbiology 7:481-488. DOI: 10.1111/j.1462-5822.2005.00506.x

Canton J, Neculai D, and Grinstein S. 2013. Scavenger receptors in homeostasis and immunity. Nature Reviews Immmunology 13:621-634. DOI: 10.1038/nri3515

Catanese MT, Graziani R, von Hahn T, Moreau M, Huby T, Paonessa G, Santini C, Luzzago A, Rice CM, Cortese R, Vitelli A, and Nicosia A. 2007. High-avidity monoclonal antibodies against the human scavenger class B type I receptor efficiently block hepatitis $\mathrm{C}$ virus infection in the presence of high-density lipoprotein. Journal of Virology 81:8063-8071. DOI: 10.1128/JVI.00193-07

Davy SK, Allemand D, and Weis VM. 2012. Cell biology of cnidarian-dinoflagellate symbiosis. Microbiology and Molecular Biology Reviews 76:229-261. DOI: 10.1128/mmbr.0501411

Detournay O, Schnitzler CE, Poole A, and Weis VM. 2012. Regulation of cnidariandinoflagellate mutualisms: Evidence that activation of a host TGF $\beta$ innate immune pathway promotes tolerance of the symbiont. Developmental and Comparative Immunology 38:525-537. DOI: 10.1016/j.dci.2012.08.008

Detournay O, and Weis VM. 2011. Role of the sphingosine rheostat in the regulation of cnidarian-dinoflagellate symbioses. Biological Bulletin 221:261-269. DOI:

Dinguirard N, and Yoshino TP. 2006. Potential role of a CD36-like class B scavenger receptor in the binding of modified low-density lipoprotein (acLDL) to the tegumental surface of 
555

556

557

558

559

560

561

562

563

564

565

566

567

568

569

570

571

572

573

574

575

576

577

578

579

580

581

582

583

584

585

586

587

Schistosoma mansoni sporocysts. Molecular Biochemical Parasitology 146:219-230.

DOI: 10.1016/j.molbiopara.2005.12.010

Faure M, and Rabourdin-Combe C. 2011. Innate immunity modulation in virus entry. Current Opinion in Virology 1:6-12. DOI: 10.1016/j.coviro.2011.05.013

Felsenstein J. 2005. SEQBOOT_-bootstrap, jackknife or permutation resampling of molecular sequence, restriction site, gene frequency or character data. University of Washington, Seattle.

Fuess LE, Pinzón C JH, Weil E, and Mydlarz LD. 2016. Associations between transcriptional changes and protein phenotypes provide insights into immune regulation in corals. Developmental \& Comparative Immunology 62:17-28. DOI: 10.1016/j.dci.2016.04.017

Gordon S. 2002. Pattern recognition receptors doubling up for the innate immune response. Cell 111:927-930. DOI: 10.1016/S0092-8674(02)01201-1

Gough PJ, and Gordon S. 2000. The role of scavenger receptors in the innate immune system. Microbes and Infection 2:305-311. DOI: 10.1016/S1286-4579(00)00297-5

Grabherr MG, Haas BJ, Yassour M, Levin JZ, Thompson DA, Amit I, Adiconis X, Fan L, Raychowdhury R, Zeng Q, Chen Z, Mauceli E, Hacohen N, Gnirke A, Rhind N, di Palma F, Birren BW, Nusbaum C, Lindblad-Toh K, Friedman N, and Regev A. 2011. Fulllength transcriptome assembly from RNA-Seq data without a reference genome. Nature Biotechnology 29:644-652. DOI: 10.1038/nbt.1883

Guindon S, Lethiec F, Duroux P, and Gascuel O. 2005. PHYML Online - a web server for fast maximum likelihood-based phylogenetic inference. Nucleic Acids Research 33:W557W559. DOI: 10.1093/nar/gki352

Hamada M, Shoguchi E, Shinzato C, Kawashima T, Miller DJ, and Satoh N. 2013. The complex NOD-like receptor repertoire of the coral Acropora digitifera includes novel domain combinations. Molecular Biology and Evolution 30:167-176. DOI: $10.1093 / \mathrm{molbev} / \mathrm{mss} 213$

Hibino T, Loza-Coll M, Messier C, Majeske AJ, Cohen AH, Terwilliger DP, Buckley KM, Brockton V, Nair SV, Berney K, Fugmann SD, Anderson MK, Pancer Z, Cameron RA, Smith LC, and Rast JP. 2006. The immune gene repertoire encoded in the purple sea urchin genome. Developmental Biology 300:349-365. DOI: 10.1016/j.ydbio.2006.08.065

Hohenester E, Sasaki T, and Timpl R. 1999. Crystal structure of a scavenger receptor cysteinerich domain sheds light on an ancient superfamily. Nature Structural Biology 6:228-232. DOI: $10.1038 / 6669$

Hsu HY, Chiu SL, Wen MH, Chen KY, and Hua KF. 2001. Ligands of macrophage scavenger receptor induce cytokine expression via differential modulation of protein kinase signaling pathways. Journal of Biological Chemistry 276:28719-28730. DOI: 10.1074/jbc.M011117200

Huang S, Yuan S, Guo L, Yu Y, Li J, and Wu T. 2008. Genomic analysis of the immune gene repertoire of amphioxus reveals extraordinary innate complexity and diversity. Genome Research 18:1112-1126. DOI: 10.1101/gr.069674.107

Janeway CA, and Medzhitov R. 2002. Innate immune recognition. Annual Review of Immunology 20:197-216. DOI:

Jimbo M, Koike K, Sakai R, Muramoto K, and Kamiya H. 2005. Cloning and characterization of a lectin from the octocoral Sinularia lochmodes. Biochemical and Biophysical Research Communications 330:157-162. DOI: 
588

589

590

591

592

593

594

595

596

597

598

599

600

601

602

603

604

605

606

607

608

609

610

611

612

613

614

615

616

617

618

619

620

621

622

623

624

625

626

627

628

629

630

631

632

Jimbo M, Yanohara T, Koike K, Koike K, Sakai R, Muramoto K, and Kamiya H. 2000. The Dgalactose-binding lectin of the octocoral Sinularia lochmodes: characterization and possible relationship to the symbiotic dinoflagellates. Comparative Biochemistry and Physiology Part B: Biochemistry and Molecular Biology 125:227-236. DOI:

Katoh K, Misawa K, Kuma K-i, and Miyata T. 2002. MAFFT: a novel method for rapid multiple sequence alignment based on fast Fourier transform. Nucleic Acids Research 30:30593066. DOI:

Kearse M, Moir R, Wilson A, Stones-Havas S, Cheung M, Sturrock S, Buxton S, Cooper A, Markowitz S, Duran C, Thierer T, Ashton B, Meintjes P, and Drummond A. 2012. Geneious Basic: an integrated and extendable desktop software platform for the organization and analysis of sequence data. Bioinformatics 28:1647-1649. DOI:

Kitchen SA, Crowder CM, Poole AZ, Weis VM, and Meyer E. 2015. De novo assembly and characterization of four anthozoan (Phylum Cnidaria) transcriptomes. G3: Genes $\mid$ Genomes $\mid$ Genetics 5:2441-2452. DOI: 10.1534/g3.115.020164

Krieger M. 1992. Molecular flypaper and atherosclerosis: structure of the macrophage scavenger receptor. Trends in Biochemical Sciences 17:141-146. DOI: 10.1016/09680004(92)90322-Z

Krieger M. 2001. Scavenger receptor class B type I is a multiligand HDL receptor that influences diverse physiologic systems. Journal of Clinical Investigation 108:793-797. DOI: 10.1172/JCI14011

Kvennefors EC, Leggat W, Kerr CC, Ainsworth TD, Hoegh-Guldberg O, and Barnes AC. 2010. Analysis of evolutionarily conserved innate immune components in coral links immunity and symbiosis. Developmental and Comparative Immunology 34:1219-1229. DOI: 10.1016/j.dci.2010.06.016

Kvennefors ECE, Leggat W, Hoegh-Guldberg O, Degnan BM, and Barnes AC. 2008. An ancient and variable mannose-binding lectin from the coral Acropora millepora binds both pathogens and symbionts. Developmental and Comparative Immunology 32:1582-1592. DOI: 10.1016/j.dci.2008.05.010

Lehnert E, Burriesci M, and Pringle J. 2012. Developing the anemone Aiptasia as a tractable model for cnidarian-dinoflagellate symbiosis: the transcriptome of aposymbiotic $A$. pallida. BMC Genomics 13:271. DOI: 10.1186/1471-2164-13-271

Lehnert EM, Mouchka ME, Burriesci MS, Gallo ND, Schwarz JA, and Pringle JR. 2014. Extensive differences in gene expression between symbiotic and aposymbiotic Cnidarians. G3: Genes|Genomes|Genetics 4:277-295. DOI: 10.1534/g3.113.009084

Li B, Lu F, Wei X, and Zhao R. 2008. Fucoidan: structure and bioactivity. Molecules 13:16711695. DOI: 10.3390/molecules13081671

Martinez VG, Moestrup SK, Holmskov U, Mollenhauer J, and Lozano F. 2011. The conserved scavenger receptor cysteine-rich superfamily in therapy and diagnosis. Pharmacological Reviews 63:967-1000. DOI: 10.1124/pr.111.004523

McFall-Ngai M, Hadfield MG, Bosch TCG, Carey HV, Domazet-Lošo T, Douglas AE, Dubilier N, Eberl G, Fukami T, Gilbert SF, Hentschel U, King N, Kjelleberg S, Knoll AH, Kremer N, Mazmanian SK, Metcalf JL, Nealson K, Pierce NE, Rawls JF, Reid A, Ruby EG, Rumpho M, Sanders JG, Tautz D, and Wernegreen JJ. 2013. Animals in a bacterial world, a new imperative for the life sciences. Proceedings of the National Academy of Sciences 110:3229-3236. DOI: 10.1073/pnas.1218525110 
633 Meyer E, and Weis VM. 2012. Study of cnidarian-algal symbiosis in the 'omics' age. Biological Bulletin 223:44-65. DOI:

635

636

637

638

639

640

641

642

643

644

645

646

647

648

649

650

651

652

653

654

655

656

657

658

659

660

661

662

663

664

665

666

667

668

669

670

671

672

673

674

675

676

677

678

Miller D, Hemmrich G, Ball E, Hayward D, Khalturin K, Funayama N, Agata K, and Bosch T. 2007. The innate immune repertoire in Cnidaria - ancestral complexity and stochastic gene loss. Genome Biology 8:R59. DOI:

Moya A, Huisman L, Ball E, Hayward D, Grasso L, Chua C, Woo H, GATTUSO JP, Forêt S, and Miller D. 2012. Whole transcriptome analysis of the coral Acropora millepora reveals complex responses to $\mathrm{CO}_{2}$-driven acidification during the initiation of calcification. Molecular Ecology 21:2440-2454. DOI: 10.1111/j.1365294X.2012.05554.X

Ndungu FM, Urban BC, Marsh K, and Langhorne J. 2005. Regulation of immune response by Plasmodium-infected red blood cells. Parasite Immunololgy 27:373-384. DOI: 10.1073/pnas.230096397

Pancer Z. 2000. Dynamic expression of multiple scavenger receptor cysteine-rich genes in coelomocytes of the purple sea urchin. Proceedings of the National Academy of Sciences 97:13156-13161. DOI: 10.1073/pnas.230096397

Pancer Z, Munkner J, Muller I, and Muller W. 1997. A novel member of an ancient superfamily: sponge (Geodia cydonium, Porifera) putative protein that features scavenger receptor cysteine-rich repeats. Gene 193:211-218. DOI: 10.1016/S0378-1119(97)00135-2

Pancer Z, Rast JP, and Davidson EH. 1999. Origins of immunity: transcription factors and homologues of effector genes of the vertebrate immune system expressed in sea urchin coelomocytes. Immunogenetics 49:773-786. DOI: doi:10.1007/s002510050551

Perez S, and Weis VM. 2006. Nitric oxide and cnidarian bleaching: An eviction notice mediates the breakdown of symbiosis. Journal of Experimental Biology 209:2804-2810. DOI:

Poole AZ, and Weis VM. 2014. TIR-domain-containing protein repertoire of nine anthozoan species reveals coral-specific expansions and uncharacterized proteins. Developmental and Comparative Immunology 46:480-488. DOI: 10.1016/j.dci.2014.06.002

Price MN, Dehal PS, and Arkin AP. 2010. FastTree 2 - Approximately Maximum-Likelihood Trees for Large Alignments. PLoS One 5:e9490. DOI: 10.1371/journal.pone.0009490

Putnam NH, Srivastava M, Hellsten U, Dirks B, Chapman J, Salamov A, Terry A, Shapiro H, Lindquist E, Kapitonov VV, Jurka J, Genikhovich G, Grigoriev IV, Lucas SM, Steele RE, Finnerty JR, Technau U, Martindale MQ, and Rokhsar DS. 2007. Sea anemone genome reveals ancestral eumetazoan gene repertoire and genomic organization. Science 317:86-94. DOI: 10.1126/science.1139158

Quevillon E, Silventoinen V, Pillai S, Harte N, Mulder N, Apweiler R, and Lopez R. 2005. InterProScan: protein domains identifier. Nucleic Acids Research 33:W116-W120. DOI: 10.1093/nar/gki442

R-Core-Team. 2012. R: A language and environment for statistical computing. R Foundation for Statistical Computing, Vienna, Austria. ISBN 3-900051-07-0, URL http://www. Rproject. org.

Rast JP, and Messier-Solek C. 2008. Marine invertebrate genome sequences and our evolving understanding of animal immunity. Biological Bulletin 214:274-283. DOI:

Rodrigues CD, Hannus M, Prudêncio M, Martin C, Gonçalves LA, Portugal S, Epiphanio S, Akinc A, Hadwiger P, Jahn-Hofmann K, Röhl I, van Gemert G-J, Franetich J-F, Luty AJF, Sauerwein R, Mazier D, Koteliansky V, Vornlocher H-P, Echeverri CJ, and Mota MM. 2008. Host scavenger receptor SR-BI plays a dual role in the establishment of 
679

680

681

682

683

684

685

686

687

688

689

690

691

692

693

694

695

696

697

698

699

700

701

702

703

704

705

706

707

708

709

710

711

712

713

714

715

716

717

718

719

720

721

722

723

malaria parasite liver infection. Cell Host \& Microbe 4:271-282. DOI:

10.1016/j.chom.2008.07.012

Rodriguez-Lanetty M, Phillips W, and Weis V. 2006. Transcriptome analysis of a cnidarian dinoflagellate mutualism reveals complex modulation of host gene expression. $B M C$ Genomics 7:23. DOI: 10.1186/1471-2164-7-23

Ryu T, Seridi L, Moitinho-Silva L, Oates M, Liew YJ, Mavromatis C, Wang X, Haywood A, Lafi FF, Kupresanin M, Sougrat R, Alzahrani MA, Giles E, Ghosheh Y, Schunter C, Baumgarten S, Berumen ML, Gao X, Aranda M, Foret S, Gough J, Voolstra CR, Hentschel U, and Ravasi T. 2016. Hologenome analysis of two marine sponges with different microbiomes. BMC Genomics 17:1-11. DOI: 10.1186/s12864-016-2501-0

Sarrias M, Grønlund J, and Padilla O. 2004. The scavenger receptor cysteine-rich (SRCR) domain: an ancient and highly conserved protein module of the innate immune system. Critical Reviews in Immunology 24:1-37. DOI: 10.1615/CritRevImmunol.v24.i1.10

Schneider DS, and Ayres JS. 2008. Two ways to survive infection: what resistance and tolerance can teach us about treating infectious diseases. Nature Reviews Immunology 8:889-895. DOI: $10.1038 /$ nri2432

Schwarz RS, Hodes-Villamar L, Fitzpatrick KA, Fain MG, Hughes AL, and Cadavid LF. 2007. A gene family of putative immune recognition molecules in the hydroid Hydractinia. Immunogenetics 59:233-246. DOI: 10.1007/s00251-006-0179-1

Shinzato C, Shoguchi E, Kawashima T, Hamada M, Hisata K, Tanaka M, Fujie M, Fujiwara M, Koyanagi R, Ikuta T, Fujiyama A, Miller DJ, and Satoh N. 2011. Using the Acropora digitifera genome to understand coral responses to environmental change. Nature 476:320-323. DOI: 10.1038/nature10249

Silverstein RL, and Febbraio M. 2009. CD36, a scavenger receptor involved in immunity, metabolism, angiogenesis, and behavior. Science Signaling 2:re3. DOI: 10.1126/scisignal.272re3

Thelen T, Hao Y, Medeiros AI, Curtis JL, Serezani CH, Kobzik L, Harris LH, and Aronoff DM. 2010. The class A scavenger receptor, macrophage receptor with collagenous structure is the major phagocytic receptor for Clostridium sordellii expressed by human decidual macrophages. Journal of Immunology 185:4328-4335. DOI: 10.4049/jimmunol.1000989

Vidal-Dupiol J, Adjeroud M, Roger E, Foure L, Duval D, Mone Y, Ferrier-Pages C, Tambutte E, Tambutte S, Zoccola D, Allemand D, and Mitta G. 2009. Coral bleaching under thermal stress: putative involvement of host/symbiont recognition mechanisms. $B M C$ Physiolology 9:14. DOI: 10.1186/1472-6793-9-14

Weis VM, and Allemand D. 2009. What determines coral health? Science 324:1153-1155. DOI: 10.1126/science. 1172540

Wood-Charlson EM, and Weis VM. 2009. The diversity of C-type lectins in the genome of a basal metazoan, Nematostella vectensis. Developmental and Comparative Immunology 33:881-889. DOI: 10.1016/j.dci.2009.01.008

Yellowlees D, Rees TAV, and Leggat W. 2008. Metabolic interactions between algal symbionts and invertebrate hosts. Plant Cell \& Environment 31:679-694. DOI: doi:10.1111/j.13653040.2008.01802.x

Yuen B, Bayes JM, and Degnan SM. 2014. The Characterization of Sponge NLRs Provides Insight into the Origin and Evolution of This Innate Immune Gene Family in Animals. Molecular Biology and Evolution 31:106-120. DOI: 10.1093/molbev/mst174 
724 Zani IA, Stephen SL, Mughal NA, Russell D, Homer-Vanniasinkam S, Wheatcroft SB, and 725 Ponnambalam S. 2015. Scavenger receptor structure and function in health and disease. Cells 4:178-201. DOI: 10.3390/cells4020178

727

728 
729 Table 1. Information on the cnidarian sequence resources used in this study. Non-symbiotic 730 refers to species that do not form symbioses with dinoflagellates. Aposymbiotic refers to species 731 that do form symbioses but the material from which the sequencing was performed did not 732 contain symbionts.

733

\begin{tabular}{|l|l|l|l|l|}
\hline \multicolumn{1}{|c|}{ Organism } & $\begin{array}{c}\text { Developmental } \\
\text { stage }\end{array}$ & Symbiotic state & \multicolumn{1}{|c|}{ Data type } & \multicolumn{1}{|c|}{ Reference } \\
\hline Nematostella vectensis & Larvae & Non-symbiotic & Genome & (Putnam et al. 2007) \\
\hline Anthopleura elegantissima & Adult & Aposymbiotic & Transcriptome & (Kitchen et al. 2015) \\
\hline Aiptasia pallida & Adult & Aposymbiotic & Transcriptome & (Lehnert et al. 2012) \\
\hline Aiptasia pallida & Adult & Aposymbiotic & Genome & (Baumgarten et al. 2015) \\
\hline Acropora digitifera & Sperm & Aposymbiotic & Genome & (Shinzato et al. 2011) \\
\hline Acropora millepora & Adult and larvae & Symbiotic & Transcriptome & (Moya et al. 2012) \\
\hline Fungia scutaria & Larvae & Aposymbiotic & Transcriptome & (Kitchen et al. 2015) \\
\hline
\end{tabular}

734 
736

737

738

739

740

741

742

743

744

745

746

747

748

749

750

751

752

753

754

755

756

757

758

759

760

761

762

763

764

765

766

767

768

769

770

771

772

773

774

775

776

\section{Figure Legends}

Figure 1. Domain architecture of vertebrate SRs relevant to this study. All SR sequences are anchored in the membrane with one or two transmembrane domains. All have very short cytoplasmic tails and extensive extracellular ligand-binding domains. SR-As contain a collagen domain(s) and can include an SRCR or a CTLD. SR-Bs have two cytoplasmic tails on either side of a CD36 domain that forms an extracellular loop. SR-Es are defined by the presence of a CTLD. SR-Is have multiple SRCR repeats and no other identifiable extracellular domains. C, carboxy terminus; CTLD, C type lectin domain; LOX1, lectin-like oxidized low density lipoprotein receptor 1; MARCO, macrophage receptor with collagenous structure; $\mathrm{N}$, amino terminus; SRCL, scavenger receptor with C-type lectin; SRCR, scavenger receptor cysteine-rich domain.

Figure 2. Domain architecture of cnidarian SR domains in the six resources searched compared to human SRs. Identified cnidarian SR-A-like and SR-E-like sequences display diverse domain architecture and include novel domain combinations not found in vertebrates. SR-I-like sequences had a varying number of SRCR repeats. A variety of SRCR-domain-containing cnidarian sequences identified did not fit the criteria of any vertebrate SR classes and are presented as SRCR + CUB domains or SRCR + trypsin domains. SR-B-like domain combinations closely resembled vertebrate SR-Bs with two transmembrane domains, two cytoplasmic tails and a CD36 domain. CTLD, $\mathrm{C}$ type lectin domain; CUB, complement $\mathrm{C} 1 \mathrm{r} / \mathrm{C} 1 \mathrm{~s}$, Uegf, BMP1; DMBT, deleted in malignant brain tumor protein; Ig, immunoglobulin; I-Set, intermediate set of immunoglobulin domain; LDL, low density lipoprotein; LOX1, lectin-like oxidized low density lipoprotein receptor 1; EGF, epidermal growth factor; MAM meprin/A5protein/PTPmu; MARCO, macrophage receptor with collagenous structure; SCARA5, scavenger receptor class A member 5; SRCL, scavenger receptor with C-type lectin; SRCR, scavenger receptor cysteine-rich domain; U-box, ubiquitin box. Human SR data taken from Canton et al (2013). (See Supplementary File 1 for sequence information.)

Figure 3. Maximum-likelihood tree of SR-Bs from across the Metazoa. The tree was constructed with the CD36 domain of each protein using FastTree v 2.1.5. Bootstrap support values were generated using SEQBOOT, values above 0.6 are displayed at nodes. The alignment, including organism names, is displayed in Figure S1.

Figure 4. Experimental colonization by S. minutum CCMP830 of aposymbiotic A. pallida treated with increasing levels of the SR inhibitor, fucoidan. Graph shows percent colonization success as measured by surface area of host gastrodermis occupied by symbionts (see Methods for details) as a function of time after inoculation. Two controls were included: FSW alone and an $18 \mathrm{~h}$ incubation in $200 \mu \mathrm{g} / \mathrm{ml}$ fucoidan in FSW followed by a $48 \mathrm{~h}$ recovery in FSW to test for fucoidan toxicity to the animals. Anemones in experimental fucoidan treatments exhibited a 
777 dose-dependent response with decreased colonization success with increasing fucoidan

778 concentrations. Bars represent means $\pm \mathrm{SD}, \mathrm{n}=3$ anemones per treatment. Asterisks indicate

779 high $(\mathrm{p}>0.999)$ posterior probability of treatment effects being different from controls under the

780 Bayesian ANOVA model.

781

782 Figure 5. Effect of SR inhibition by fucoidan on immune stimulation in symbiotic A. pallida.

783 Immune stimulation of animals was elicited by incubation in $1 \mu \mathrm{g} / \mathrm{ml}$ LPS overnight prior to the 784 experiment. Immune stimulation was measured by quantifying DAF-FM DA, a probe for the 785 presence NO, itself a marker for immune stress. Graph shows MFI of DAF-FM DA in tentacles 786 in response to incubation in increasing concentrations of fucoidan. Animals exhibited a 787 significant dose-dependent response to fucoidan (Bayesian generalized linear mixed model, $\mathrm{P}<$ 788 0.0001), with increasing NO production with increasing SR inhibition by fucoidan. Bars 789 represent means $\pm \mathrm{SD} ; \mathrm{n}=3$ anemones. Inset: representative confocal images of tentacles 790 incubated in FSW only and $800 \mu \mathrm{g} / \mathrm{ml}$ fucoidan. DAF-FM DA (green) symbiont 791 autofluorescence (red).

792

793 


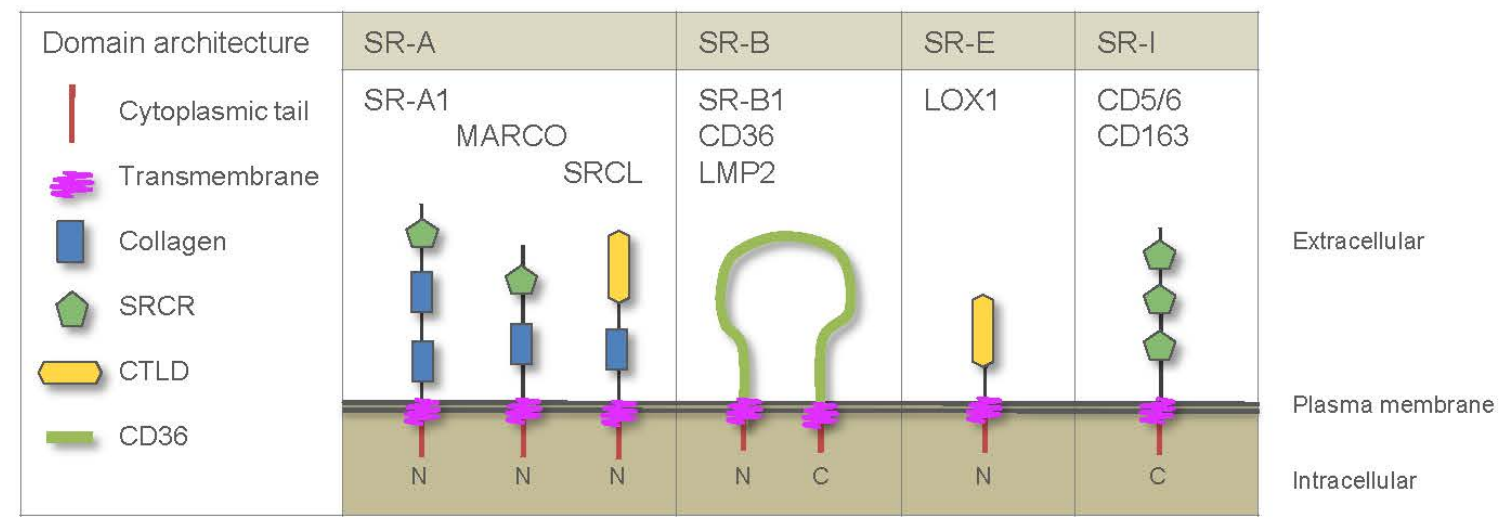

794

795

Figure 1

796 


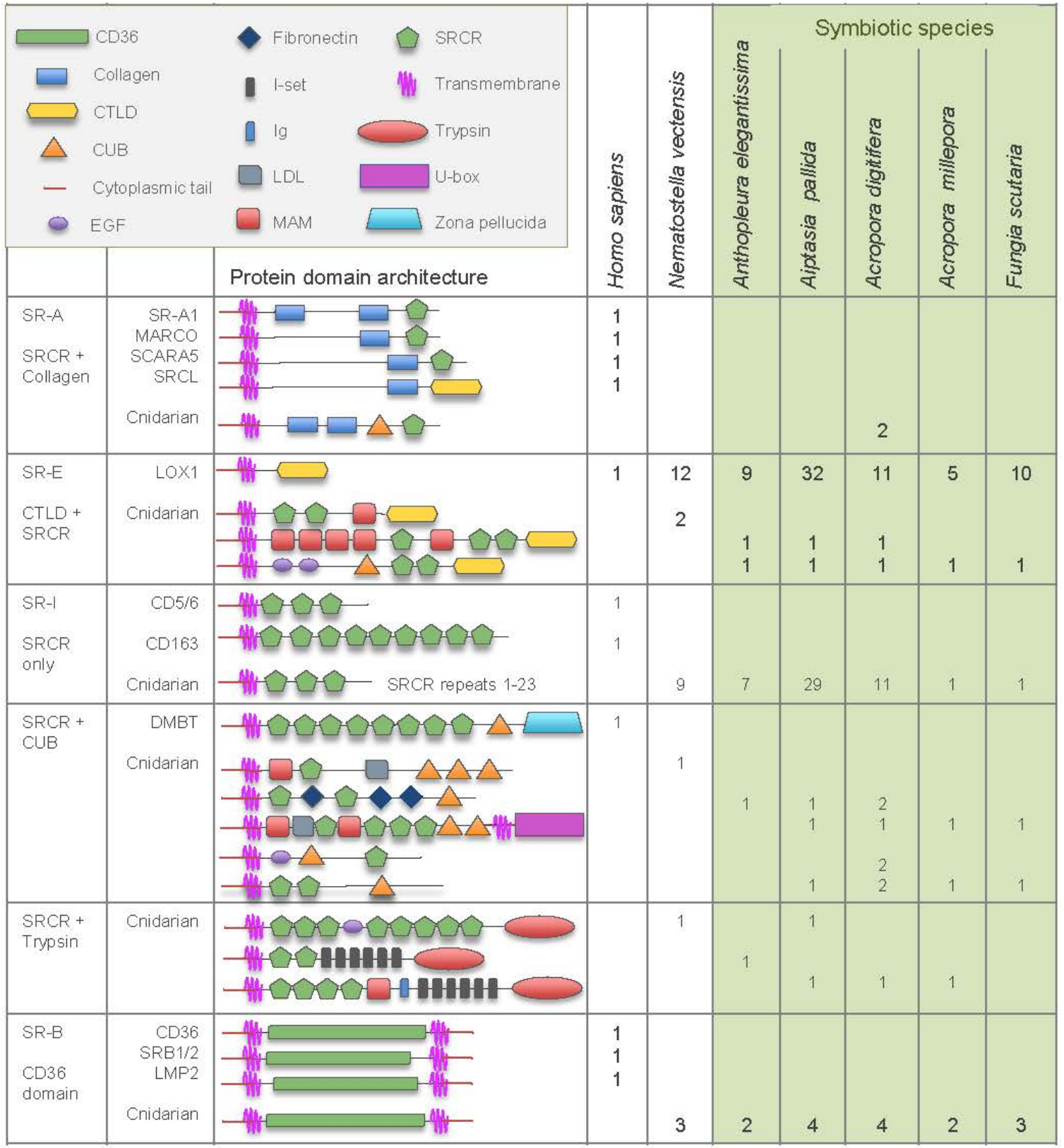

Figure 2 


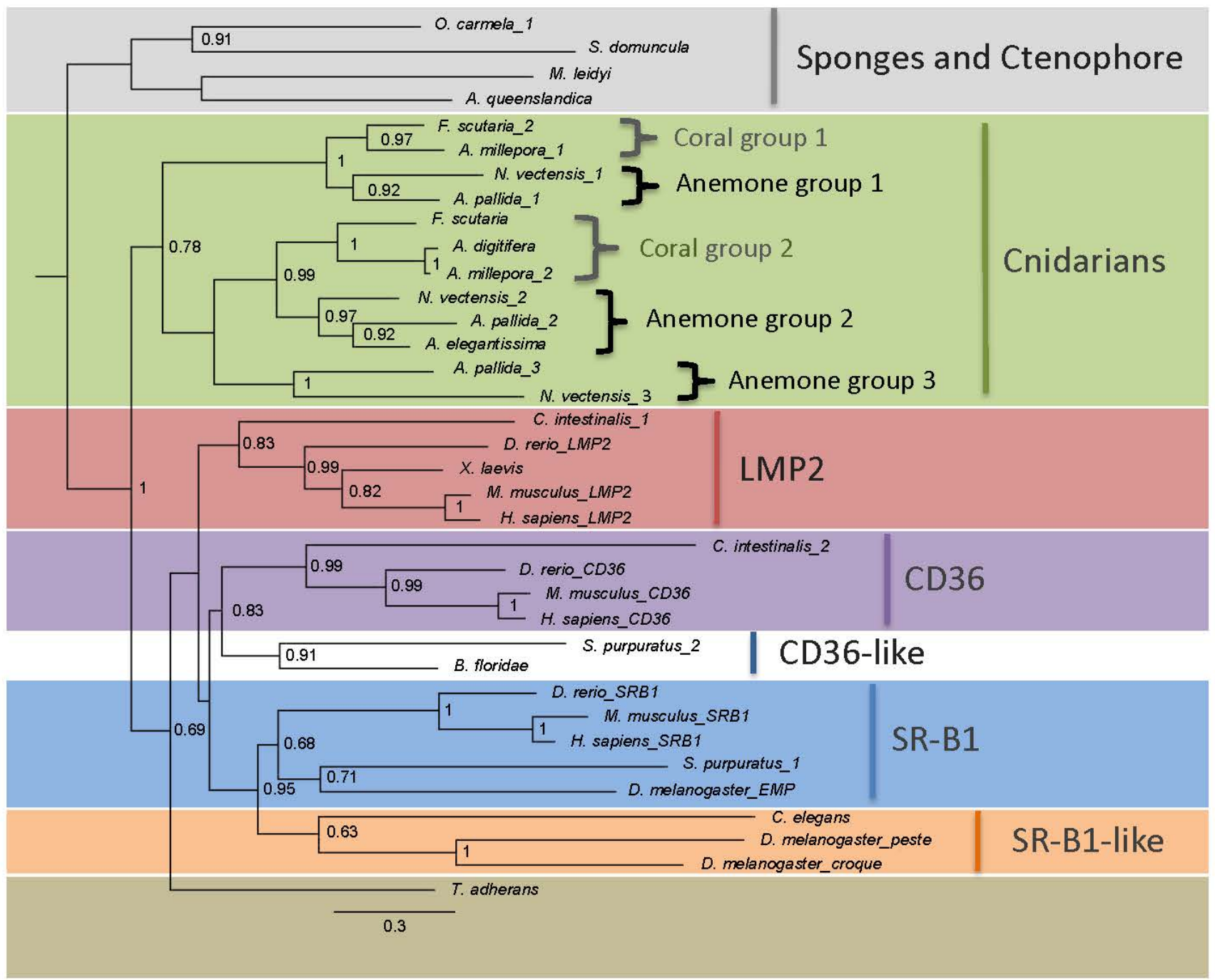

$801 \quad$ Figure 3

802 


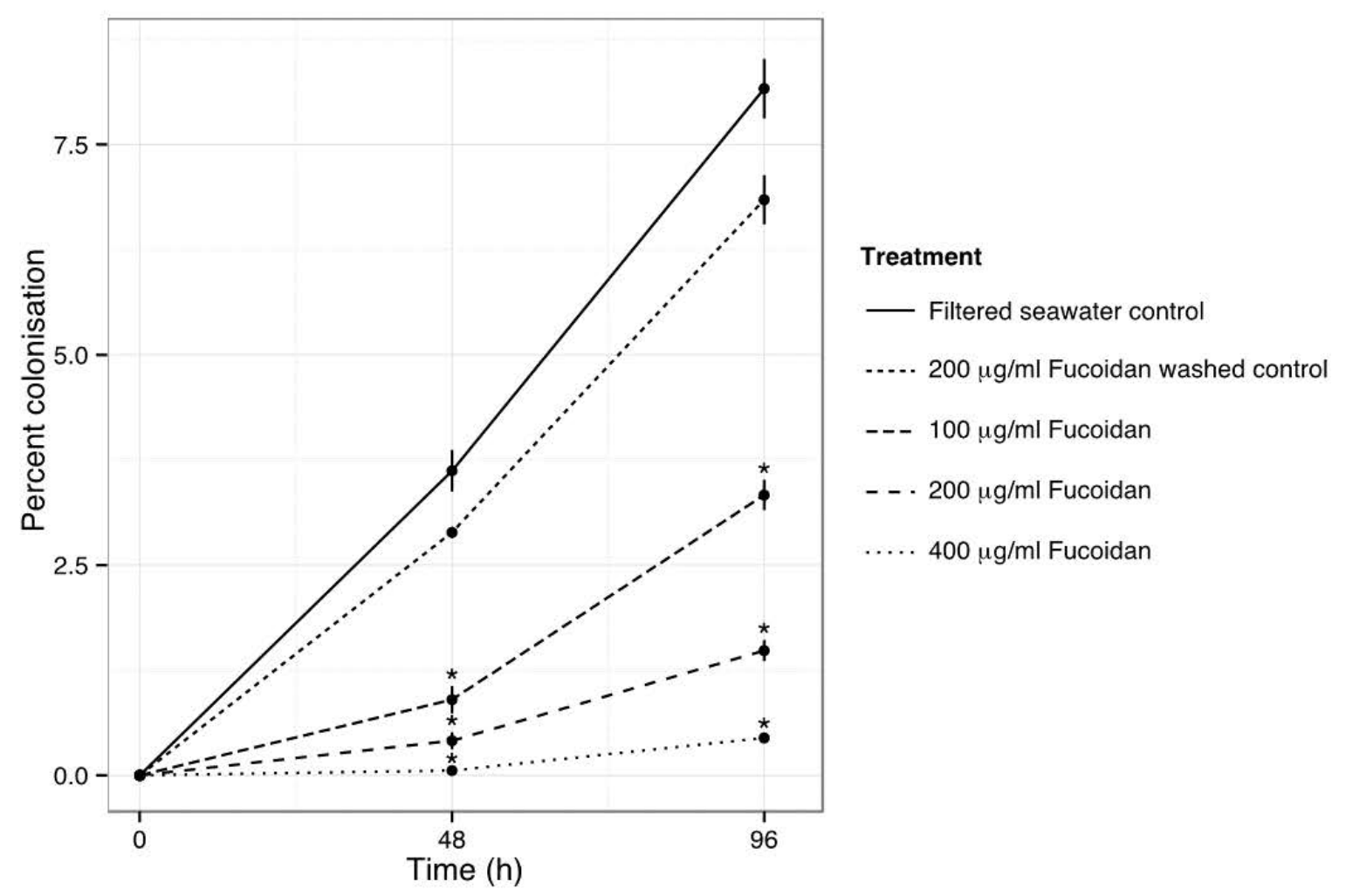

803

804

Figure 4

805 


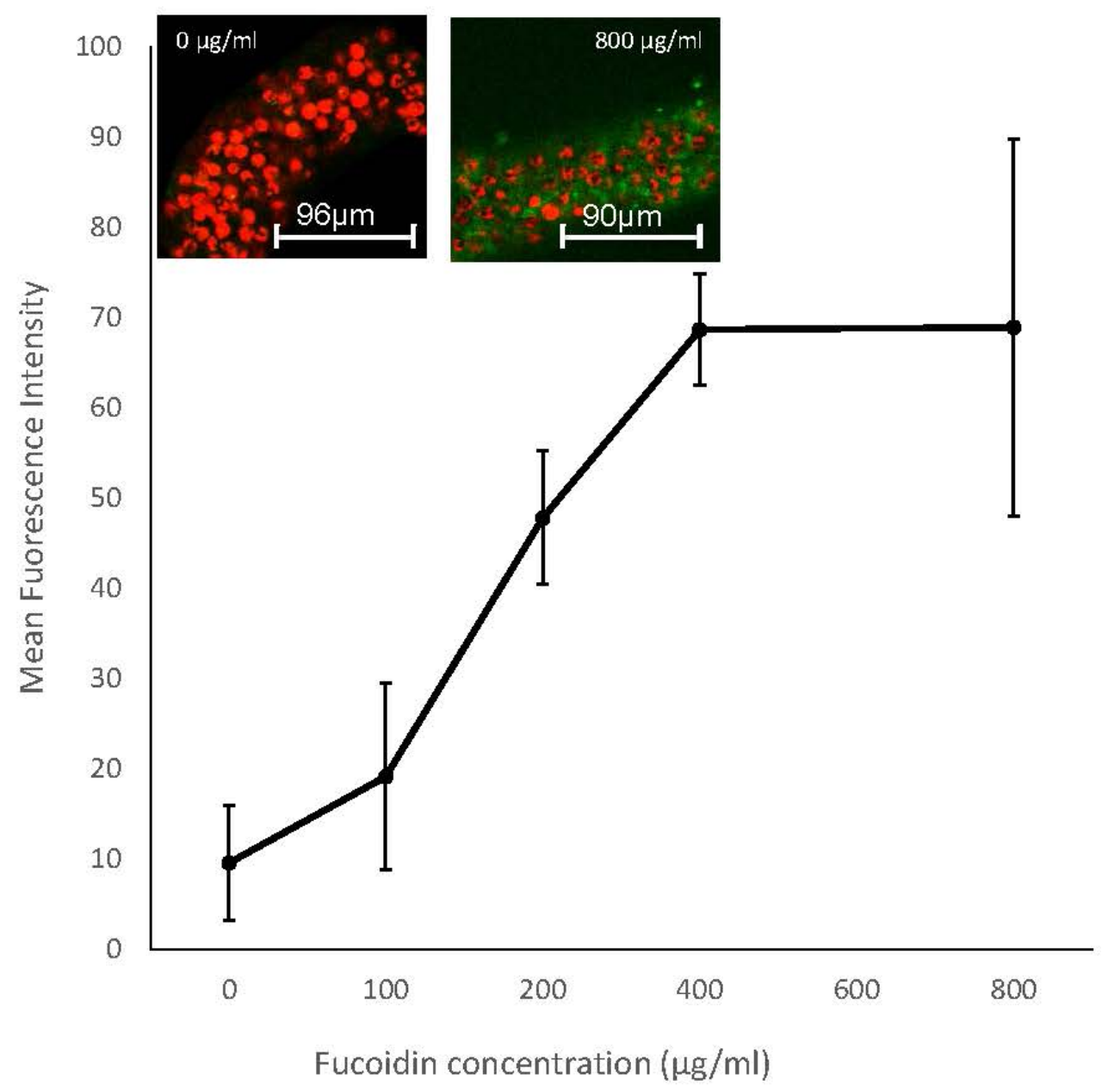

806

807 Figure 5 